\title{
Study on Designing for Inner Grid of Offshore Wind Farm
}

\author{
Je-Seok Shin, Wook-Won Kim, and Jin-O Kim
}

\begin{abstract}
Wind power is one of the widely used renewable resources and it is connected to power system steadily. In recent years, wind power is developed in the form of large-scale wind farm at offshore, which is composed of dozens or hundreds of wind turbines. In inner grid of wind farm, wind turbines are connected to each other through cable, and there are a wide variety of configurations depending on how to connect wind turbines. Due to difficult and expensive construction activity at sea, the problem to connect optimally wind turbines is very important. In order to solve the problem, this paper introduces a methodology based on the k-clustering algorithm, minimum spanning tree (MSP) algorithm and local search method. K-clustering is applied to divide wind turbines into k-groups, and MSP algorithm is used to link wind turbines in each group with the objective that total length of cables is minimized. Optimal configuration is determined by local search method which explores diverse combinations depending on the number of groups and the number of wind turbines in each group. The case studies show that the proposed methodology can be utilized usefully for designing inner grid of offshore wind farm.
\end{abstract}

Index Terms - Offshore wind farm, inner grid, k-clustering algorithm, minimum spanning tree, local search method.

\section{INTRODUCTION}

Wind power is a widely used renewable energy and is increased steadily. In particular, it is being developed as large-scale wind farm as the related technology and economics are advanced [1]. In order to gather more wind energy and to avoid environment problems and construction concerns such as securing the installation site, the recent wind power is developed at offshore. Compared with onshore wind farm, offshore wind farm (OWF) has some advantages, and it has also crucial disadvantages which are more expensive investment and maintenance costs caused by the difficulty of geographical access. Namely, since OWF operators have a considerable burden on investment, it is important to design optimal configuration for wind farm. Therefore, this paper investigates for optimal configuration of inner grid among components making up offshore wind farm. In the methodology, K-clustering algorithm divides wind turbines in inner grid into k-groups and then Minimum Spanning Tree (MSP) algorithm links wind turbines to each other based on the objective that total length of cables used for the connection of wind turbines in each group is minimized [2], [3]. Using local search method [4], the exploration is performed about diverse combinations made by the number of groups and the number of wind turbines belonging to each group. Alternatives generated by K-clustering and MST

Manuscript received June 13, 2014; revised July 15, 2014.

The authors are with the Electrical Engineering Department, Hanyang University, Seoul, Korea (e-mail: \{kjboy, neocruser, jokim\}@ hanyang.ac.kr). algorithms are evaluated in terms of total length of cables or total investment cost, and optimal configuration is finally selected. The rest of the paper is organized as follows; in Section II, typical compositions of OWF and layouts of inner grid are described. In Section III, the methodology including k-clustering, MST algorithms and local search method is introduced. In Section IV, brief case studies are performed to demonstrate the methodology. Finally, Section V contains a conclusion of this paper.

\section{INNER GRID OF OFFSHORE WIND FARM}

Typical large scaled offshore wind farm is composed of four components which are wind turbines, inner grid, offshore substation and external grid as show in Fig. 1 [1].

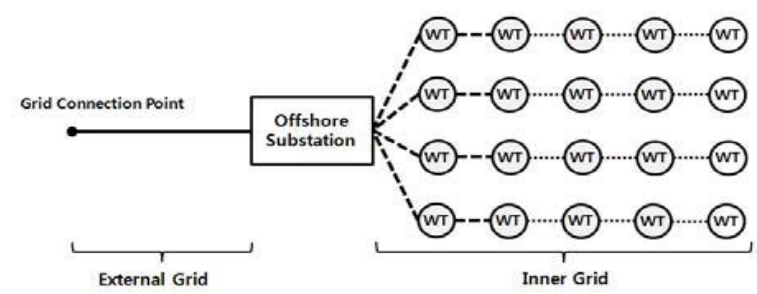

Fig. 1. Typical composition of offshore wind farm.

The locations of wind turbines and substation are restricted to places where wind turbines and substation are easy to construct and particularly wind turbines can gather more wind energy. However, inner grid which aggregates power generated at all wind turbines, is has diverse configurations according to how to connect wind turbines. Factors affecting the configurations are as follows [5].

- the voltage of inner grid

- transfer capacity of cables

- the number of feeders(wind turbines per feeder)

- the layout for inner grid

In particular, typical layouts are shown in the following Fig. 2, Fig. 3, and Fig. 4 [6].

In this paper, ring layout is not considered since it is natural that effect of improved reliability by the ring layouts is generally very small enough to be ignored compared with investment cost for them [5], [6].

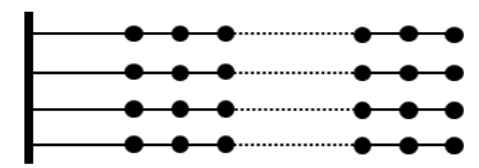

Fig. 2 Typical radial layout.

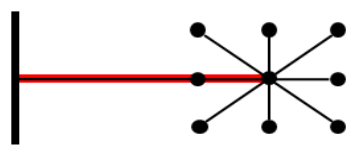

Fig. 3. Typical star layout. 


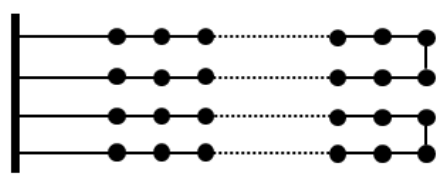

Fig. 4. Typical ring layout.

\section{Methodology FOR DeSIGN OF INNER GRID}

\section{A. K-Clustering Algorithm}

$\mathrm{K}$-clustering algorithm divides wind turbines in inner grid into k-groups, there are two methods according to criteria for dividing them [2]. One uses distances between an arbitrary centroid and wind turbines, and the other one uses radial angles of wind turbines on the offshore substation. The brief procedures for two methods are illustrated using the following example as shown in Fig. 5.

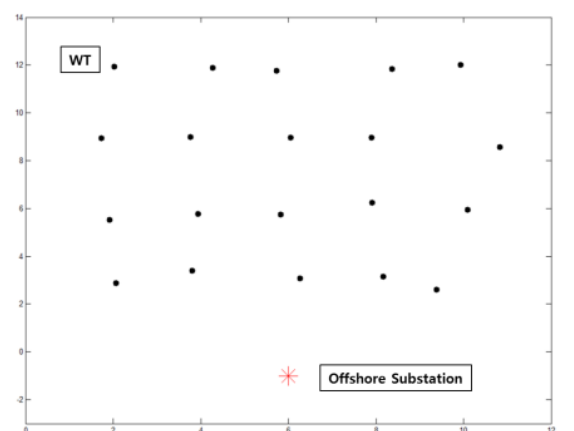

Fig. 5. Example for locations of WT and offshore substation.

Criterion1) Distance between Centroid and WTs

1) Determine the number of groups $(K)$

2) Assume arbitrary positions of k-centroids

3) Find the closest centroid for each wind turbine and then, assign each wind turbine the corresponding group number.

4) Calculate position of new centroid as the middle point of wind turbines belonging to same group.

5) Until the position of centroids is not changed, iterate steps 3 ) and 4).

The Fig. 6 shows the result applying the criterion 1, where the number of groups is three and the numbers of wind turbines in each group are 6, 7 and 7, respectively. It seems that wind turbines are near to each other in each group, so that wind turbines in the group can be linked closely. However, some of groups are located far away from offshore substation. This fact can result in increase of investment cost since the length of the thickest cables to transfer all power generated in the group are longer.

Criterion2) Radial Angle of WT on the basis of offshore substation

1) Determine the number of groups $(K)$ and the numbers of wind turbines belonging to individual groups.

2) Calculate the radial angle of wind turbines on the basis of offshore substation.

3) Rotate a boundary line clockwise from the left side of offshore substation until the number of wind turbines divided by the line is as much as the value in the corresponding group. And then, assign the wind turbines a number of the corresponding group.
4) Until all wind turbine are sorted, repeat step 3)

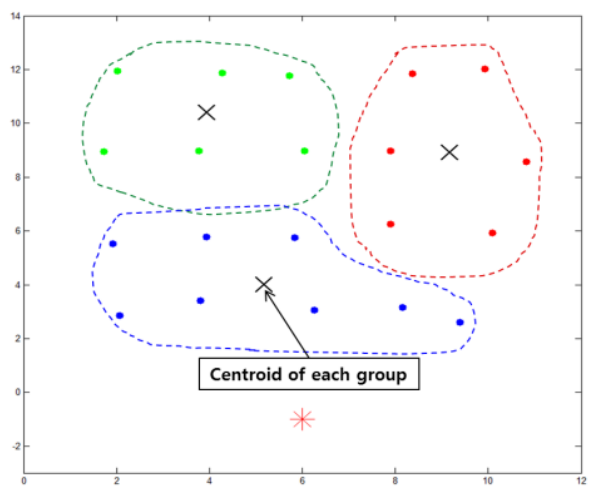

Fig. 6. Result of clustering wind turbines using criterion 1.

The Fig. 7 shows the result applying the criterion 2, where the number of groups is three and the numbers of wind turbines in each group are 6, 7 and 7, respectively.

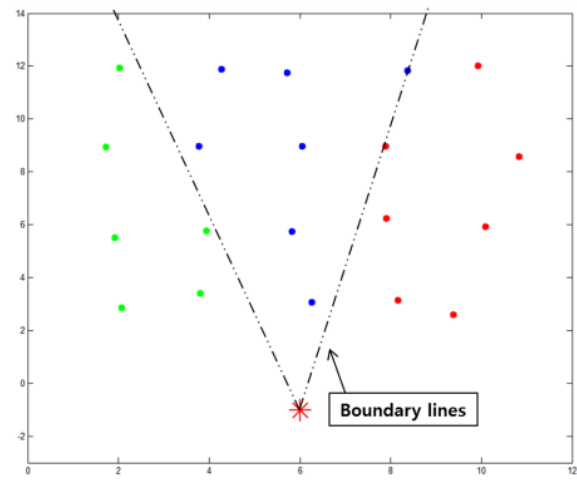

Fig. 7. Result of clustering wind turbines using criterion 2.

Compared with the formal result using criterion 1 , the result using criterion 2 shows that all groups can be linked with offshore substation more closely. Although the superiority of the two criteria is different depending on locations of wind turbines and offshore substation, criterion 2 having the characteristic of shorter linkage with offshore substation is considered in this paper.

\section{B. Minimum Spanning Tree Algorithm}

After dividing wind turbines into k-groups, wind turbines in each group is connected each other. MST is applied to connect wind turbines by the shortest length of cables [3]. A brief procedure for MST is as follows.

1) Select the closest wind turbine in the group to offshore substation, and then the selected wind turbine is defined as start point of MST algorithm.

2) Calculate distances between the stating wind turbine and other remaining wind turbines, and then, select wind turbine with the shortest distance and connect with the starting wind turbine.

3) Calculate distances between the selected wind turbine and remaining ones, and then select wind turbine with the shortest distance from the accumulated results in the formal steps and the current step, and connect two wind turbines with the shortest distance.

4) Until the all wind turbine are linked, repeat step 4).

Result of applying MST algorithm to Fig. 7 is represented in the following Fig. 8. 


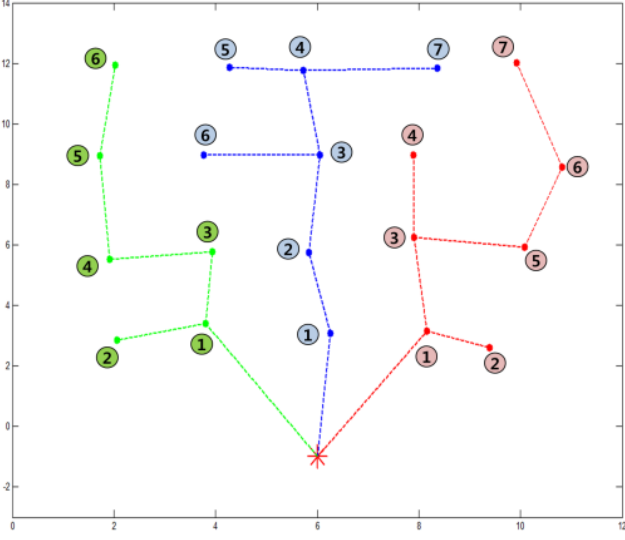

Fig. 8. Result applying MST algorithm.

In Fig. 8, numbers in each group represent the connecting order of each wind turbine.

\section{Local Search Method}

The number of groups in inner grid is affected by the capacity of available cable and, its minimum value can be determined by the maximum number of wind turbine to be accommodated by the thickest cable $\left(N W_{\text {Cable }}^{\text {Max }}\right)$. This is represented by the following (1).

$$
\left[\frac{N W_{\text {Inner }}}{N W_{\text {Cable }}^{\text {Max }}}\right] \leq k
$$

where, $N W_{\text {Inner }}$ is the total number of wind turbines in inner grid.

Information for the number of wind turbines in each group can be represented by (2), (3).

$$
\begin{gathered}
\left(N W_{1}, N W_{2}, \ldots, N W_{k}\right) \\
\sum_{i=1}^{k} N W_{i}=N W_{\text {Inner }}, \quad 0<N W_{i} \leq N W_{\text {Cable }}^{\text {Max }}
\end{gathered}
$$

A brief procedure for the local search is illustrated through the example of 4-groups with condition that the maximum number of wind turbines in each group is 7, as follows [6].

1) Assume initial information as the base alternative. Initial information: $(5,5,5,5)$

2) Generate alternatives based on the initial information. If there is change on the number of wind turbines in a group, the value for the next group is revised. For example, if the number of the first group is changed to 6 from 5 , then, the number of the second group is changed to 4 from 5 in order to satisfy the condition expressed by (3). As a result, alternatives adjacent to the initial information can be obtained as shown in Table I.

3) Perform the formal described two algorithms on the all alternatives.

4) Evaluate alternatives in terms of total length or total investment cost. Select the alternative with minimum total length or total investment cost, and then the selected alternative is the base one in the next exploration.

5) Until the more improved alternative is not found, repeat steps 2)-4).

TABLE I: ALTERNATIVES TO BE EXPLORED BY LOCAL SEARCH the Number of Wind Turbines

\begin{tabular}{cllll} 
No. Group & 1 & 2 & 3 & 4 \\
\hline Base one & 5 & 5 & 5 & 5 \\
Alternative 1 & 6 & 4 & 5 & 5 \\
Alternative 2 & 4 & 6 & 5 & 5 \\
Alternative 3 & 5 & 6 & 4 & 5 \\
Alternative 4 & 5 & 4 & 6 & 5 \\
Alternative 5 & 5 & 5 & 6 & 4 \\
Alternative 6 & 5 & 5 & 4 & 6 \\
\hline \hline
\end{tabular}

\section{CASE STUdy}

In addition to examples used to illustrate the methodology, the case study is performed continuously on the diverse number of groups. Re-mentioning about the condition for the case study, inner grid of wind farm is composed of 20 wind turbines, and locations of wind turbines are tabularized in Table II and are depicted in Fig. 5. Location of offshore

\begin{tabular}{|c|c|c|}
\hline \multirow[b]{2}{*}{ No. WT } & \multicolumn{2}{|c|}{ Location } \\
\hline & $X$ & $\mathrm{Y}$ \\
\hline 1 & 2.07 & 2.86 \\
\hline 2 & 1.92 & 5.52 \\
\hline 3 & 1.73 & 8.94 \\
\hline 4 & 2.02 & 11.94 \\
\hline 5 & 3.81 & 3.40 \\
\hline 6 & 3.94 & 5.77 \\
\hline 7 & 3.77 & 8.98 \\
\hline 8 & 4.28 & 11.87 \\
\hline 9 & 6.26 & 3.07 \\
\hline 10 & 5.83 & 5.75 \\
\hline 11 & 6.05 & 8.97 \\
\hline 12 & 5.73 & 11.76 \\
\hline 13 & 8.16 & 3.16 \\
\hline 14 & 7.91 & 6.25 \\
\hline 15 & 7.90 & 8.97 \\
\hline 16 & 8.37 & 11.84 \\
\hline 17 & 9.39 & 2.61 \\
\hline 18 & 10.09 & 5.94 \\
\hline 19 & 10.83 & 8.58 \\
\hline 20 & 9.93 & 12.01 \\
\hline
\end{tabular}
substation is assumed at $(6,-1)$. The data for cables are represented in Table III.

TABLE III: DATA FOR CABLES

\begin{tabular}{ccccc}
\hline \hline Types of Cable & 1 & 2 & 3 & 4 \\
\hline $\begin{array}{c}\text { Cross-Sectional } \\
\text { Area } \\
{[\mathrm{mm} 2]}\end{array}$ & 70 & 185 & 400 & 500 \\
$\begin{array}{c}\text { No. of } \\
\text { Connectable WT } \\
\text { Investment Cost } \\
{\left[\$ 10^{5} / \mathrm{km}\right]}\end{array}$ & 2 & 4 & 6 & 7 \\
\hline \hline
\end{tabular}

From Fig. 5, it is reasonable to consider $3 \sim 5$ as the range 
for the number of groups since 2-groups don't accommodate all wind turbines even if the thickest cable is used. On the other hand, in the case of 6-groups, at least one wind turbine in the second row should be linked with offshore substation, so that it results in the excessive total length of cables.

In order to compare results using two criteria for the optimum, the results of 3-groups are represented in Table IV.

TABLE IV: RESULTS FOR 3-GROUPS

\begin{tabular}{cccccc}
\hline \hline No. Group & 1 & 2 & 3 & $\begin{array}{c}\text { Length } \\
{[\mathrm{km}]}\end{array}$ & $\begin{array}{c}\text { Investment Cost } \\
{\left[\$ 10^{5} / \mathrm{km}\right]}\end{array}$ \\
\hline Alternative 1 & 6 & 7 & 7 & 57.14 & 803.46 \\
Alternative 2 & 7 & 7 & 6 & 55.82 & 816.24 \\
\hline \hline
\end{tabular}

As shown in Table IV, alternative 2 minimizes the total length of cables as $55.82 \mathrm{~km}$ and alternative 1 minimizes the investment cost as $\$ 803.46 \times 10^{5}$. From this result, it is indicated that the minimum total length must not guarantee minimum investment cost. The Fig. 9 and Fig. 10 show the layouts of alternative 1 and 2, respectively, where the number of group is named from the left side of the figure.

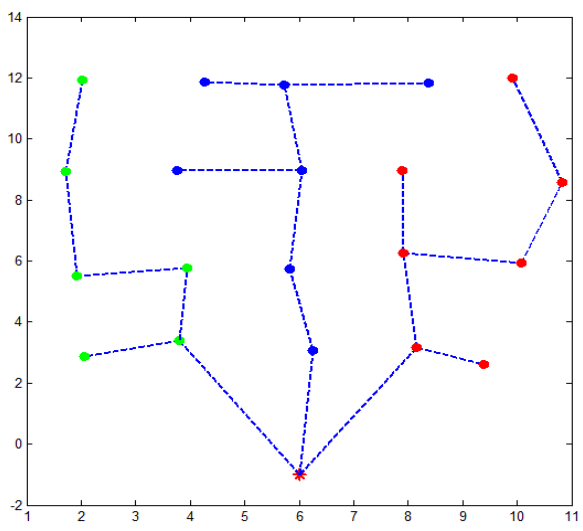

Fig. 9. Layout of alternative 1.

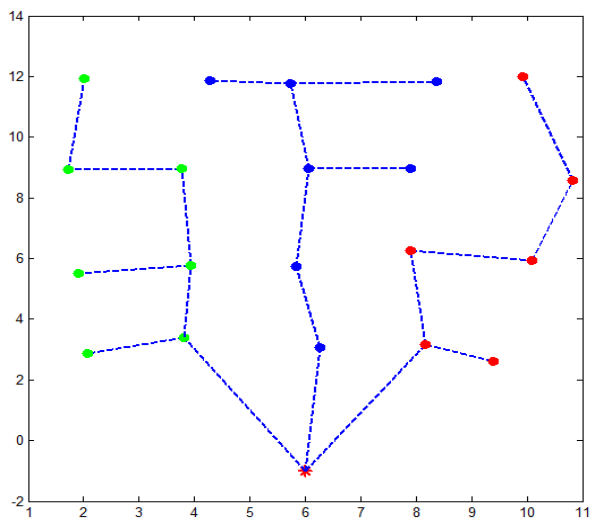

Fig. 10. Layout of alternative 2 .

For 4-groups and 5-groups, the results with the minimum cost are tabularized in Table $\mathrm{V}$.

TABLE V: RESULTS FOR 4-GROUPS AND 5-GROUPS

\begin{tabular}{cccccccc}
\hline \hline No. Group & 1 & 2 & 3 & 4 & 5 & $\begin{array}{c}\text { Length } \\
{[\mathrm{km}]}\end{array}$ & $\begin{array}{c}\text { Investment Cost } \\
{\left[\$ 10^{5} / \mathrm{km}\right]}\end{array}$ \\
\hline 4-Groups & 4 & 6 & 7 & 3 & - & 58.79 & 819.58 \\
5-Groups & 4 & 4 & 5 & 4 & 3 & 65.87 & 876.85 \\
\hline \hline
\end{tabular}

Fig. 11 and Fig. 12 show the layout of 4-Groups and 5-Groups, respectively.

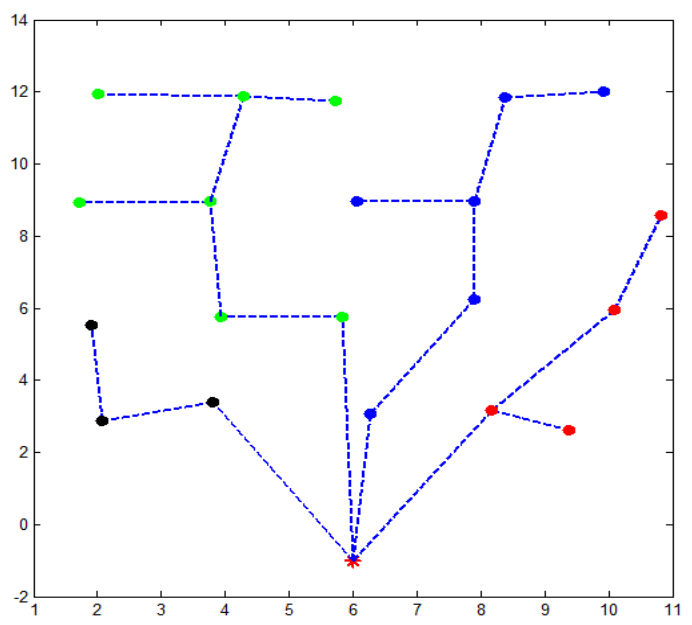

Fig. 11. Layout of 4-groups.

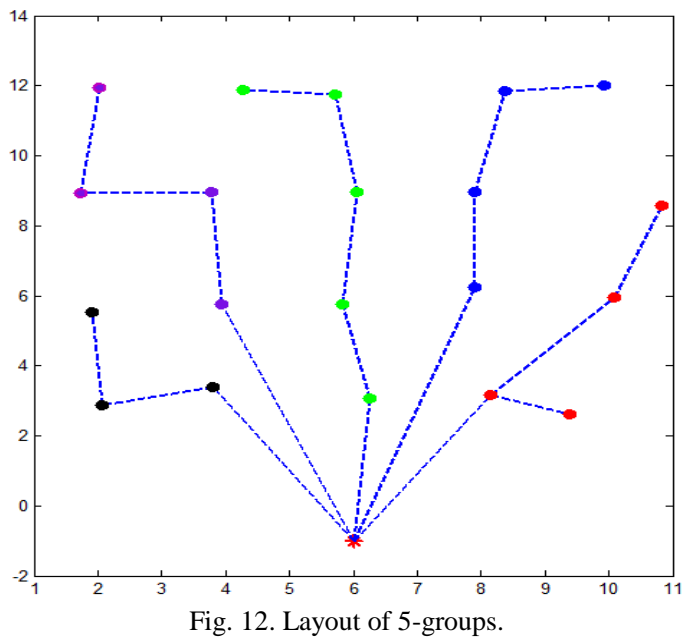

As the number of groups is increased, the layout can be configured using thinner cables and thus it can result in less investment cost. However, the cables linking wind turbine and offshore substation also lengthen, the investment cost is increased.

\section{CONCLUSION}

This paper introduces the methodology to design optimal layout for inner grid. Dozens or hundreds of wind turbines are divided into several groups by k-clustering algorithm with the radial angle criterion. And then, wind turbines in each group are connected through minimum spanning tree algorithm under the objective which minimizes the total length of cables. The result of layout can be evaluated in terms of total length of cables or total investment cost. The case studies show that evaluation using total investment cost is reasonable. In order to explore more wide alternatives, local search is applied until more improved layout is found. Finally, optimal layout is determined as one with minimum total investment cost. In order to determine optimal layout of inner grid comprehensively, the next steps of research focus on the improved methodology which considers power loss cost and the reliability evaluation. 


\section{REFERENCES}

[1] T. Ackermann, Wind Power in Power Systems, 1st ed. New York: Wiley, 2005.

[2] S. Dutta and T. J. Overbye, "Optimal wind farm collector system topology design considering total trenching length," IEEE Trans. on Sustainable Energy, vol. 3, pp. 339-348, July 2012.

[3] Lecture 6: minimum spanning trees and prim's algorithm. [Online]. Available:

http://www.cs.umd.edu/ meesh/420/Notes/MountNotes/lecture6-mst prim.pdf

[4] A. Arabali, M. Ghofrani, M. Etezadi-Amoli, and M. S. Fadali, "Stochastic performance assessment and sizing for a hybrid power system of solar/wind/energy storage," IEEE Trans. on Sustainable Energy, vol. 5, pp. 363-371, April 2014.

[5] J. S. Shin, W. W. Kim, I. S. Bae, and J. O. Kim, "A study on reliability assessment for offshore wind farm configurations," presented at SMARTGREENS 2014, Barcelona, Spain, 03-04 April, 2014.

[6] P. Djapic and G. Strbac, "Cost Benefit methodology for optimal design of offshore transmission systems," Centre for Sustainable Electricity and Distributed Generation, July, 2008.

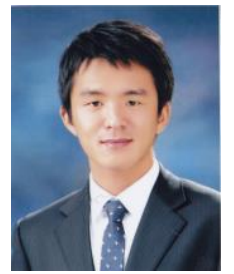

Je-Seok Shin received the B.S. degrees in electrical engineering from Hanyang University, Seoul, Korea, in 2010. He is currently a Ph.D. candidate in Department of Electrical Engineering of Hanyang University. His research interests are integration of wind farm, operation of power system with wind power and power system planning and reliability evaluation.

Wook-Won Kim received the B.S. and M.S. degrees in electrical engineering from Hanyang University, Seoul, South Korea, in 2008 and 2011, respectively. He is currently a Ph.D. candidate in Department of Electrical Engineering of Hanyang University. His research interests are configuration of renewable energy in smart grid, optimal operation for renewable energy and reliability evaluation.

Jin-O Kim received the B.S. and M.S. degrees in electrical engineering from Seoul National University, Seoul, Korea, in 1980 and 1983, respectively, and the Ph.D. degree from Texas A\&M University, College Station, in 1991. $\mathrm{He}$ is presently a professor with the Department of Electrical and Control Engineering, Hanyang University, Seoul. His research interests are power system reliability, planning, and power economics and reliability centered maintenance and demand response. 